Abanico Veterinario. Enero-Diciembre 2021; 11:1-16. http://dx.doi.org/10.21929/abavet2021.29

Artículo Original. Recibido: 09/03/2021. Aceptado: 14/06/2021. Publicado: 21/06/2021. Clave: e2021-21.

\title{
Detección molecular de Ehrlichia canis y Anaplasma phagocytophilum y alteraciones hematológicas de perros infectados
}

\author{
Molecular detection of Ehrlichia canis and Anaplasma phagocytophilum and \\ hematological changes of infected dogs
}

\section{Octavio Merino-Charrez ID, Valeria Badillo-Moreno ${ }^{\mathrm{ID}}$, Jorge Loredo-Osti ${ }^{\mathrm{ID}}$, Hugo Barrios-García ID, Verónica Carvajal-de-la-Fuente ${ }^{*}$ ID}

Facultad de Medicina Veterinaria y Zootecnia "Dr. Norberto Treviño Zapata". Universidad Autónoma de Tamaulipas, km 5, Carretera Victoria-Mante, Ciudad Victoria, Tamaulipas, CP 87000, México. *Autor para correspondencia: Verónica Carvajal-de-la-Fuente. Carretera Victoria-Mante km 5. CP. 87000, Ciudad Victoria, Tamaulipas, México. omerino@docentes.uat.edu.mx, valeria.badillo@live.com, jloredo@docentes.uat.edu.mx, hbarrios@docentes.uat.edu.mx,vcarvajal@docentes.uat.edu.mx

\section{RESUMEN}

Las ehrlichiosis y anaplasmosis canina son enfermedades transmitidas por garrapatas, provocadas por bacterias del género Ehrlichia y Anaplasma. Debido a sus múltiples manifestaciones clínicas, su diagnóstico es un reto para el veterinario. La distribución de estos hemoparásitos incluye áreas donde su principal vector, Rhipicephalus sanguineus está presente. Este estudio fue diseñado para determinar la presencia Ehrlichia canis y Anaplasma phagocytophilum, así como los factores asociados y hallazgos hematológicos comunes en perros de la zona centro de Tamaulipas. Se evaluaron, a través de PCR, 384 muestras de sangre provenientes de animales de diferentes clínicas veterinarias y un refugio. El análisis de datos se realizó con la prueba Chi cuadrada con un nivel de significancia de 0.05 . Los resultados muestran que, del total de muestras $103(26.8 \%)$ resultaron positivas a E. canis, mientras que para A. phagocytophilum no se detectó ningún caso. No se observó asociación significativa con relación al sexo, raza, ni lugar de procedencia ( $p>0.05)$, a diferencia de la edad, donde se encontró mayor prevalencia de $E$. canis para adultos (15-84 meses) $(p<0.05)$. En relación con el hematocrito, conteo de plaquetas, proteínas plasmáticas totales, conteo y diferencial leucocitario, no existió diferencias significativas ( $p>0.05$ ).

Palabras claves: Ehrlichia canis, Anaplasma phagocytophilum, PCR, valores hematológicos.

\begin{abstract}
Ehrlichiosis and anaplasmosis are tick-borne diseases caused by bacteria of the genera Ehrlichia and Anaplasma. Since clinical manifestations are varied and nonspecific, the diagnosis in clinical practice, remains a challenge for veterinarians. Furthermore, the distribution of these infections includes areas where its tick vector, Rhipicephalus sanguineus is present. This study was designed to evaluate the prevalence and factors associated with the presence of Ehrlichia canis and Anaplasma phagocytophilum in dogs from the central area of Tamaulipas. PCR screened 384 canine blood samples obtained from different veterinary clinics and a shelter. The data were analyzed using the Chi-square test ( $P$ level $<0.05$ for statistical significance). The results showed that $103(26.8 \%)$ out of 384 samples were positive for $E$. canis, while $A$. phagocytophilum was not detected. Statistical analysis did not show relationship between $E$. canis and variables like gender, breed, and origin ( $P>0.05)$. Nonetheless, there was a statistically significant difference between infected adult dogs (15-84 months) compared to other age groups evaluated $(p<0.05)$. Regarding hematocrit, platelets count, plasma protein, total and differential white blood cells counts, none of these parameters were significantly different $(P>0.05)$.

Keywords: Ehrlichia canis, Anaplasma phagocytophilum, PCR, hematological findings.
\end{abstract}




\section{INTRODUCCIÓN}

La ehrlichiosis y anaplasmosis son enfermedades de gran importancia tanto para la salud veterinaria como pública, ya que son responsables de enfermedades como la Ehrlichiosis Monocítica y Anaplasmosis Granulocítica Humana (Vieira et al., 2013; Farhan 2015; Rodríguez-Vivas et al., 2019). Son provocadas por bacterias intracelulares obligadas Gram negativas conocidas como Ehrlichia spp. y Anaplasma spp. respectivamente (Harrus y Waner 2011; Stuen et al., 2013). A nivel mundial, los casos se han incrementado considerablemente en los últimos años principalmente en zonas tropicales y subtropicales donde proliferan las garrapatas vectoras (Rhipicephalus sanguineus e Ixodes spp) (Beugnet y Chalvet-Monfray 2013; Irwin 2014; Little et al., 2014; Battilani et al., 2017). Debido a la cercanía cada vez mayor de las personas con sus mascotas, la probabilidad de picaduras por estos ectoparásitos aumenta considerablemente propiciando a que estas infecciones se están convirtiendo en zoonosis reemergentes (Bhadesiya y Modi 2015; Ismail y McBride 2017).

En México, la Ehrlichiosis canina se reportó por primera vez en 1996, desde entonces el número de casos se ha incrementado considerablemente (Maggi y Krämer 2019); no obstante, el diagnóstico, en muchas ocasiones se basa en signos clínicos sin realizar pruebas de laboratorio que corroboren directa o indirectamente su presencia. El diagnóstico definitivo se centra en técnicas microscópicas; sin embargo, estos métodos tienen baja sensibilidad y especificidad en pacientes con una baja bacteremia, lo cual evita establecer la terapéutica adecuada (Harrus y Waner 2011; Allison y Little 2013). En respuesta a esto, la Reacción en Cadena de la Polimerasa (PCR) surge como una importante herramienta de apoyo a los métodos de diagnóstico convencionales (Almazan et al., 2016; Cetinkaya et al., 2016; de la Fuente et al., 2017).

El estado de Tamaulipas por su ubicación geográfica cuenta con características idóneas que favorecen el desarrollo de garrapatas vectoras de estas enfermedades (TinocoGracia et al., 2009); sin embargo, se desconoce la verdadera magnitud de este problema. Por lo tanto, el principal objetivo de esta investigación fue determinar la presencia de $E$. canis y $A$. phagocytophilum, a través de PCR, en perros naturalmente infectados en la zona centro de Tamaulipas; así como evaluar algunos factores asociados a la presencia de estas enfermedades.

\section{MATERIAL Y MÉTODOS}

\section{Área de estudio}

El presente trabajo se realizó con muestras sanguíneas de perros remitidas (durante el periodo marzo de 2020 a marzo de 2021) al Laboratorio de Parasitología y Análisis Clínicos de la Facultad de Medicina Veterinaria y Zootecnia "Dr. Norberto Treviño Zapata", perteneciente a la Universidad Autónoma de Tamaulipas. Asimismo, con muestras provenientes de varias clínicas veterinaria particulares de la capital de Tamaulipas y algunos municipios aledaños. 


\section{Población de estudio}

Se utilizó un muestreo no probabilístico, en el cual se analizaron las muestras de pacientes que se remitieron con los siguientes criterios de inclusión: 1) ser del estado de Tamaulipas (zona centro), 2) presentar signos clínicos relacionados con hemoparásitos (fiebre, diarrea, uveítis, petequias, epistaxis, trastornos osteoarticulares, respiratorios, reproductivos y neurológicos), 3) presentar o haber estado en contacto con garrapatas y 4) tener el consentimiento del propietario de la mascota. El tamaño de muestra fue de 384 animales, que es el tamaño de muestra mínima que se obtiene de la fórmula de (n) para proporciones de poblaciones infinitas, ya que no existe un padrón de población canina en la zona a evaluar (Wayne y Chad 2013). Todos los perros fueron manejados de acuerdo con las normas oficiales de bienestar animal establecido por el Comité de Bioética de la Facultad de Medicina Veterinaria y Zootecnia de la Universidad Autónoma de Tamaulipas.

\section{Colección de muestras}

Se obtuvo un mínimo de $3 \mathrm{ml}$ de sangre por punción venosa (vena cefálica), los cuales fueron transferidos rápidamente a un tubo (BD Vacutainer ${ }^{\circledR}$ ) con anticoagulante EDTA $\mathrm{K}_{2}$ (ácido etilendiaminotetraacético potásico). Las muestras se mantuvieron en refrigeración $\left(8^{\circ} \mathrm{C}\right)$ por no más de 24 horas antes de ser procesada para su evaluación hematológica. Se guardó una alícuota de sangre en viales de $1.5 \mathrm{ml}$ y se almacenó a $-20{ }^{\circ} \mathrm{C}$ para su posterior extracción de ADN y prueba de PCR. En todos los casos se registró la edad, sexo, raza y estación del año en que se tomó la muestra de los individuos estudiados.

\section{Análisis hematológico}

La determinación de los parámetros hematológicos se realizó de forma inmediata, sin que pasaran más de 4 horas de haber obtenido la sangre para evitar alteraciones morfológicas de las células. Las muestras fueron analizadas en un equipo automatizado (Auto Hematology Analyzer, MINDRAY, BC-2800 Vet; Shenzhen, China). Para la determinación de las proteínas plasmáticas se utilizó el método del microhematocrito, utilizando tubos capilares sin heparina; los cuales se llenaron con 3/4 partes con sangre, se sellaron y centrifugados (centrífuga KHT-410E Kendal Import S.A.C Gemmy Taiwan) a $11,500 \mathrm{rpm}$ durante $5 \mathrm{~min}$. El plasma obtenido fue colocado en un refractómetro (American Optical) y se obtuvo el total de proteínas. Se realizó el conteo de diferencial leucocitario de manera manual. El primero consistió en valorar y contar en un frotis sanguíneo (teñido con Diff-Quik ${ }^{\mathrm{TM}}$ ) 100 células nucleadas y así obtener el recuento porcentual de los diferentes leucocitos: neutrófilos, eosinófilos, linfocitos, monocitos y basófilos. Para determinar si existía la presencia de anemia se tomó en cuenta el valor del hematocrito, el cual fue categorizado en 2 grupos, con y sin presencia de anemia. El conteo de plaquetas y proteínas totales fueron divididos en 2 grupos, animales con y sin trombocitopenia y con y sin presencia de hiperproteinemia respectivamente. El total de 
leucocitos, así como sus distintas poblaciones fueron agrupadas como conteos normales, altos y disminuidos.

\section{Identificación de hemoparásitos por microscopía}

Para la búsqueda de hemoparásitos por microscopía se elaboraron frotis sanguíneos, los cuales fueron fijados con metanol durante 5 minutos y teñidos con solución Giemsa al $10 \%$ durante $15 \mathrm{~min}$. Posteriormente se evaluaron al microscopio con el objetivo de inmersión (100x) múltiples áreas aleatorias de la monocapa y cola del frotis; aquí se buscó la presencia de mórulas (agregados citoplasmáticos de color basofílico) o cualquier otro cuerpo de inclusión compatible con hemoparásitos (Dulmer et al., 2001).

\section{ANÁLISIS MOLECULAR}

\section{Obtención de ácidos nucleicos}

De las alícuotas de sangre con EDTA almacenadas se realizaron extracciones de ADN mediante el kit comercial de extracción y purificación de ADN (Wizard® Genomic DNA Purification-Promega), de acuerdo con los protocolos establecidos por la compañía. EI ADN total extraído fue cuantificado, utilizando un espectrofotómetro (NanoDrop2000®, Thermo Scientific, Waltham, MA, Estados Unidos) y conservado a $-20{ }^{\circ} \mathrm{C}$ hasta su posterior uso en las pruebas de PCR.

\section{Reacción en Cadena de la Polimerasa}

Para el análisis molecular se amplificó una región del gen GltA (utilizado para la identificación de rickettsias que codifica para la enzima citrato sintasa) para $E$. canis y una región del gen MSP4 (complejo principal de superficie) para A. phagocytophilum. Se utilizó el kit GoTaq ${ }^{\circledR}$ Green Master Mix (Promega, Madison, WI USA. Cat. Num: M7122) de acuerdo a los protocolos establecidos por la empresa. Para esto se utilizó $21 \mu \mathrm{l}$ de solución del kit, $1 \mu \mathrm{l}$ del cebador sentido, $1 \mu \mathrm{l}$ del cebador antisentido y $2 \mu \mathrm{l}$ del ADN de cada muestra para alcanzar un volumen final de $25 \mu \mathrm{l}$. Posteriormente las muestras fueron amplificadas en el termociclador (Applied Biosystems ${ }^{\mathrm{TM}}$ Num:2720) con el protocolo de amplificación que se muestra en la tabla 1. Los productos amplificados se analizaron mediante electroforesis en gel de agarosa al 2\% en $600 \mathrm{ml}$ de TAE Buffer, $1 \mathrm{X}$ (Promega, Madison, WI USA. Cat. Num: V4271) a $120 \mathrm{~V}$ por 40 minutos utilizando el colorante de ácidos nucleicos Diamond Nucleic Acid Dye (Promega, Madison, WI USA. Cat.Num: H1181) y posteriormente visualizando bajo luz UV del transiluminador UVP (Productos Ultravioleta, Inc., California, USA. Cat. Num: TFM-30). Se utilizó como control positivo fragmentos de ADN de longitudes conocidas ( $E$. canis, $200 \mathrm{pb} ; A$. phagocytophilum: $980 \mathrm{pb}$ ) y un marcador de peso molecular de 100 bp DNA Ladder (Promega, Madison, WI USA. Cat. Num: G210A) como referencia. 
Tabla 1. Secuencia de los oligonucleótidos utilizados para cada patógeno, protocolo de amplificación y tamaño del amplificado

\begin{tabular}{|c|c|c|c|c|}
\hline $\begin{array}{c}\text { Organismo y gen } \\
\text { diana }\end{array}$ & Secuencia de oligonucleótidos (5'-3') & $\begin{array}{l}\text { Programa de } \\
\text { amplificación }\end{array}$ & $\begin{array}{l}\text { Tamaño del } \\
\text { amplificado } \\
\text { (pb) }\end{array}$ & Referencia \\
\hline $\begin{array}{l}\text { E. canis } \\
\text { (GltA) }\end{array}$ & $\begin{array}{l}\text { E. canis Fw ATAAACACGCTGACTTTACTGTTCC } \\
\text { E canis Rev GTGATGAGATAGAGCGCAGTACC }\end{array}$ & $\begin{array}{l}95^{\circ} \mathrm{C} \text { por } 5 \mathrm{~min} \\
94^{\circ} \mathrm{C} \text { por } 30 \mathrm{~s} \\
60^{\circ} \mathrm{C} \text { por } 30 \mathrm{~s} \\
72^{\circ} \mathrm{C} \text { por } 1 \mathrm{~min} \\
72^{\circ} \mathrm{C} \text { por } 7 \mathrm{~min} \\
35 \text { ciclos }\end{array}$ & 200 & $\begin{array}{l}\text { Stich et al., } \\
\quad 2002\end{array}$ \\
\hline $\begin{array}{l}\text { A. phagocytophilum } \\
\text { (Msp4) }\end{array}$ & $\begin{array}{c}\text { MSP4AP5 ATGAATTACAGAGAATTGCTTGTAGG } \\
\text { MSP4AP3 } \\
\text { TTAATTGAAAGCAAATCTTGCTCCTATG }\end{array}$ & $\begin{array}{l}94^{\circ} \mathrm{C} \text { por } 5 \mathrm{~min} \\
94^{\circ} \mathrm{C} \text { por } 30 \mathrm{~s} \\
50^{\circ} \mathrm{C} \text { por } 30 \mathrm{~s} \\
72^{\circ} \mathrm{C} \text { por } 30 \mathrm{~s} \\
72^{\circ} \mathrm{C} \text { por } 7 \mathrm{~min} \\
35 \text { ciclos }\end{array}$ & 849 & $\begin{array}{c}\text { Yousefi et al., } \\
2019\end{array}$ \\
\hline
\end{tabular}

\section{Análisis estadísticos}

Se representaron las frecuencias absolutas y porcentajes de casos positivos y hallazgos hematológicos. El grado de asociación entre la presencia de patógenos y las variables evaluadas (sexo, edad, raza, época del año y parámetros hematológicos); se analizaron mediante la prueba de independencia de Chi cuadrada con un nivel de significancia de 0.05; utilizando el programa estadístico MedCalc. V. 7.0.

\section{RESULTADOS}

\section{Presencia de E. canis y A. phagocytophilum}

Del número total de muestras analizadas (384) durante el periodo de estudio, 103 resultaron positivos a $E$. canis (frecuencia de $26.8 \%$ ) mediante la técnica de PCR, donde se amplificó el gen GltA con un tamaño molecular esperado de 200 pb, como se puede observar en la figura 1. De los frotis sanguíneos evaluados se logró identificar $E$. canis en sólo 41 de las muestras evaluadas (10.7\%). Las mórulas se observaron en el citoplasma de linfocitos y monocitos como estructuras redondas, con un tamaño entre 4 a $6 \mu \mathrm{m}$ de diámetro que se tiñeron fuertemente de un color basofílico; como se muestra en la figura 2. Por otro lado, ninguno de los perros evaluados por PCR o evaluación de frotis resultó positivo para A. phagocytophilum (figura 3). 


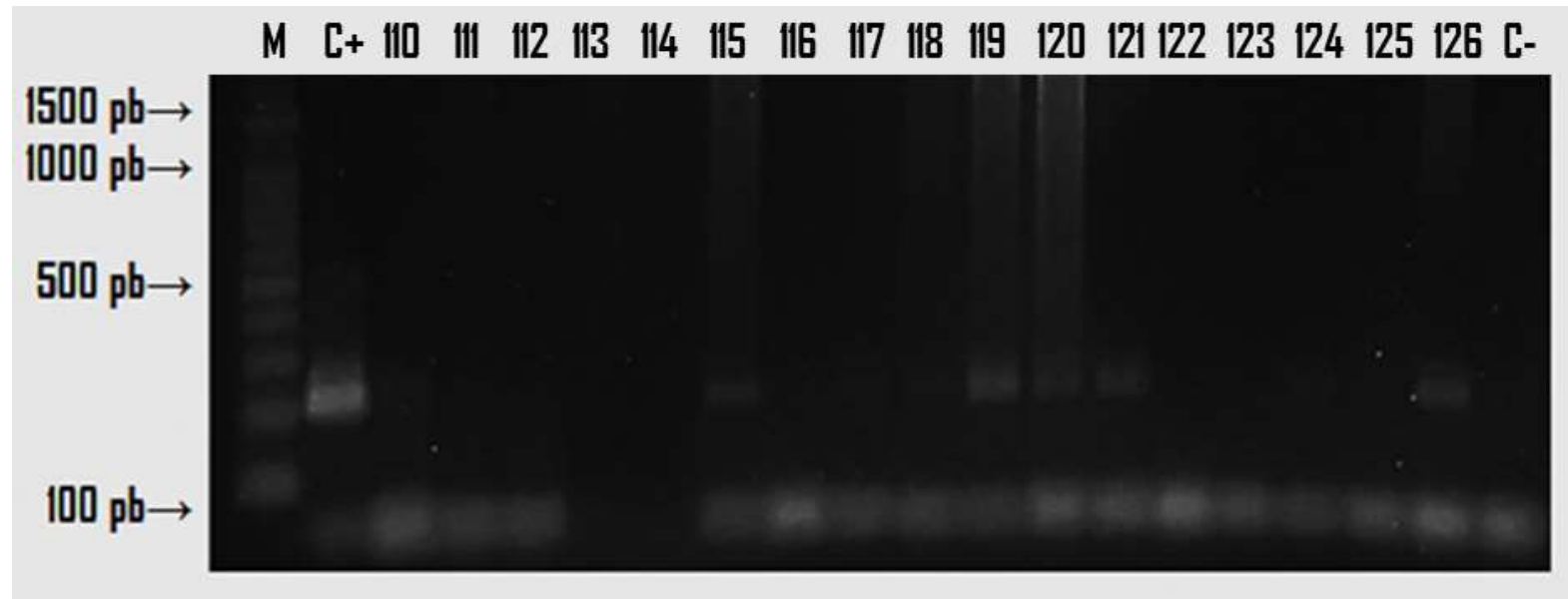

(M) Marcador de peso molecular, $\left(\mathrm{C}_{+}\right)$control positivo con peso molecular de $200 \mathrm{pb},(115,117,118,119$, $120,121,126)$ muestras positivas, (C-) control negativo con agua bidestilada. Gel de agarosa al $2 \%$, teñido con Diamond.

Figura 1. Amplificación por PCR de E. canis en muestras de sangre tomada de caninos

\section{Características de la población canina}

Se evaluaron 192 hembras (50\%) y 192 machos (50\%), con edades comprendidas entre 3 meses hasta 20 años. Los resultados observados nos muestran que E. canis no distingue entre género; ya que dentro del grupo de infectados los porcentajes de hembras (29.7) y machos (24.0) no fueron estadísticamente significativos ( $p>0.05)$. Al evaluar la relación entre la edad del perro (cachorros, adultos o seniors) y el porcentaje de positivos a $E$. canis, se determinó que existe relación significativa entre ambas variables, donde la condición de adulto ( 1 a 7 años) está relacionada con la presencia de la enfermedad $(p<0.05)$ (tabla 2).

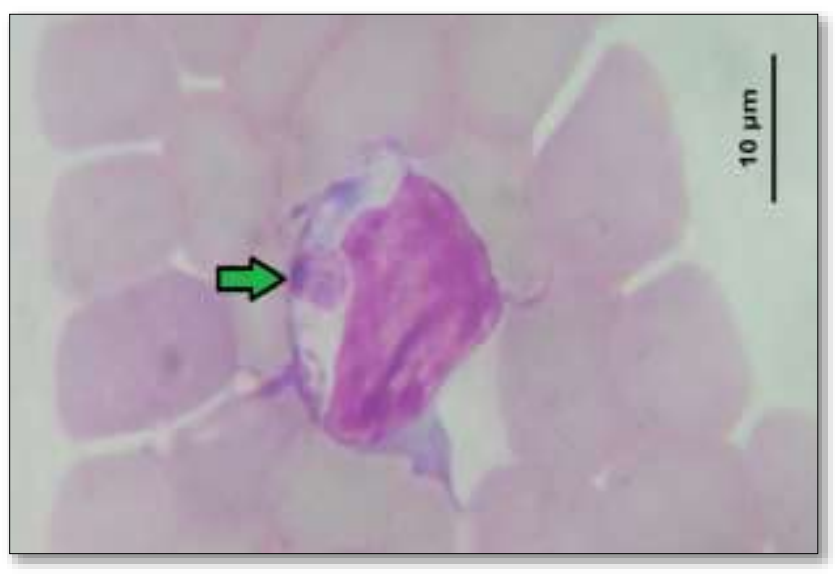

Figura 2. Linfocito en sangre periférica de un canino infectado con una mórula de $E$. canis (flecha). Tinción de Giemsa $10 \%$ 


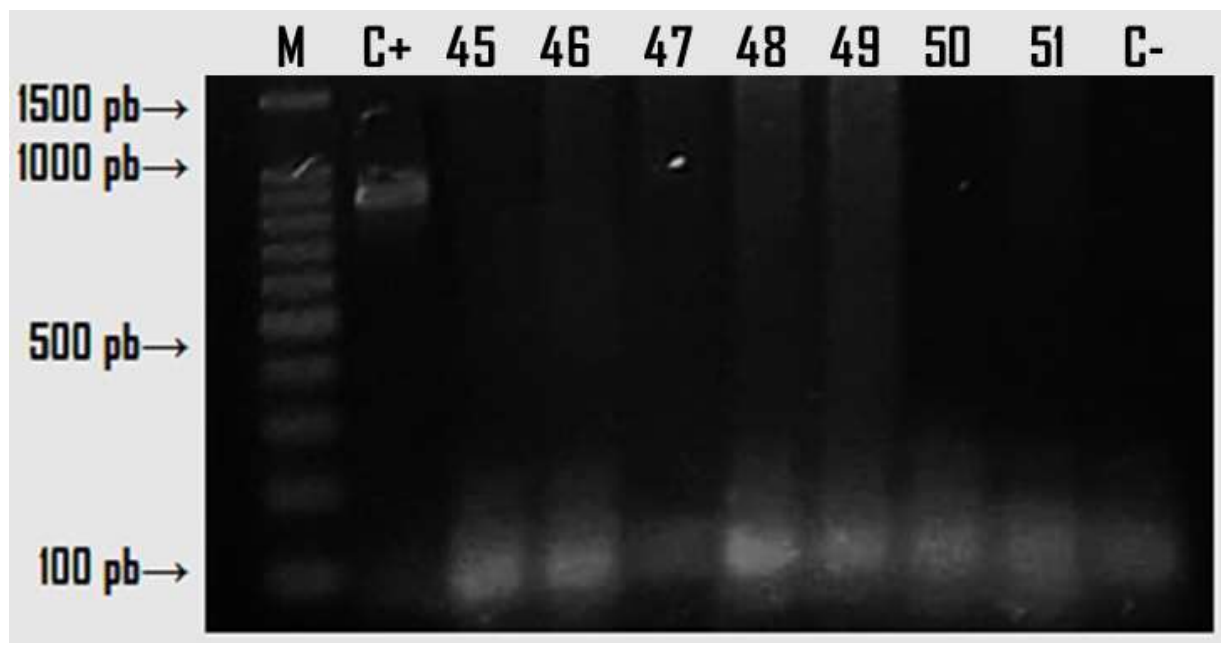

(M) Marcador de peso molecular, $\left(\mathrm{C}_{+}\right)$control positivo con peso molecular de $849 \mathrm{pb},(45-51)$ muestras negativas, (C-) control negativo con agua bidestilada. Gel de agarosa al $2 \%$, teñido con Diamond.

Figura 3. Amplificación por PCR de A. phagocytophilum en muestras de sangre tomada de caninos

Los perros de raza representaron el $81 \%$ (311/384) de la población de estudio y los mestizos constituyeron el 19\% (73/384); sin embargo, la prueba de independencia de chi cuadrada no encontró diferencias estadísticas significativas entre el resultado positivo a $E$. canis, en relación con los grupos raciales definidos y los mestizos ( $p>0.05$ ) (tabla 2). De la misma manera, no se encontraron diferencias significativas entre la presencia de Ehrlichiosis con la época del año (tabla 2).

Tabla 2. Frecuencias y porcentajes de positivos y negativos a $E$. canis agrupados por característica del animal y época del año

\begin{tabular}{|c|c|c|c|c|c|}
\hline \multicolumn{5}{|c|}{ Presencia de E. canis } & \multirow[t]{3}{*}{ Valor de $p$} \\
\hline \multirow[t]{2}{*}{ Variable } & \multicolumn{2}{|c|}{ Positivo } & \multicolumn{2}{|c|}{ Negativo } & \\
\hline & Frecuencia & $\%$ & Frecuencia & $\%$ & \\
\hline Sexo & & & & & 0.205 \\
\hline Macho & 42 & 24.0 & 146 & 76.0 & \\
\hline Hembra & 57 & 29.7 & 135 & 70.3 & \\
\hline Edad & & & & & 0.016 \\
\hline Cachorro (0-12 meses) & 16 & 22.2 & 56 & 77.8 & \\
\hline Adulto (1 a 7 años) & 69 & 32.5 & 143 & 67.5 & \\
\hline Senior (>7 años) & 18 & 18.0 & 82 & 82.0 & \\
\hline Raza & & & & & 0.981 \\
\hline Mestizo & 19 & 26.0 & 54 & 74.0 & \\
\hline De raza & 84 & 27.0 & 227 & 73.0 & \\
\hline Época del año & & & & & 0.816 \\
\hline Primavera-Verano & 71 & 26.3 & 199 & 73.7 & \\
\hline Otoño-Invierno & 32 & 28.1 & 82 & 71.9 & \\
\hline
\end{tabular}




\section{Variables hematológicas}

En relación con los hallazgos de laboratorio para los perros positivos a E. canis no existió diferencias significativas en aquellos que presentaban anemia, trombocitopenia 0 hiperproteinemia comparado con los animales negativos quienes muchos de ellos presentaban porcentajes parecidos al grupo de infectado ( $p>0.05$ ). Por otro lado, sí se encontraron diferencias significativas para algunos parámetros de la serie blanca, como el recuento total de leucocitos y neutrófilos $(p<0.05)$. Sin embargo, para estos analitos, la mayor cantidad de perros infectados con Ehrlichia fueron los que resultaron con valores dentro de los rangos de referencia, en comparación con los animales que resultaron negativos en donde se muestra una gran cantidad de perros con leucocitosis o neutrofilia. Para el resto de los parámetros hematológicos evaluados la prueba estadística no encontró diferencias significativas ( $p>0.05$ ), como se observa en la tabla 3.

\section{DISCUSIÓN}

Hoy en día, la ehrlichiosis y anaplasmosis canina han ganado mayor importancia a nivel mundial, lo cual se atribuye principalmente a que su vector (Rhipicephalus sanguineus) es considerado como la especie de garrapata con mayor distribución geográfica (Aguiar et al., 2007; Parola et al., 2013; Cabezas-Cruz et al., 2019). En este trabajo de investigación se encontró que del número total de perros evaluados (384), 103 resultaron positivos a $E$. canis $(26.8 \%)$ mediante la técnica de PCR y solamente $41(10.7 \%)$ a través de la evaluación del frotis sanguíneo. Esta discrepancia de los 2 métodos utilizados es similar a lo reportado por Happi et al., (2018), quienes de un total de116 muestras de perros sólo $10.3 \%$ resultaron positivas por microscopía, comparada con la técnica de PCR donde se obtuvo 42 resultados positivos (36.2\%). Estos resultados eran de esperarse, ya que aunque el diagnóstico por visualización microscópica de las típicas inclusiones intracelulares o mórulas dentro del citoplasma de los monocitos o linfocitos en extendidos de sangre periférica (figura 3), ha sido de gran importancia; esta técnica presenta ciertas desventajas, como la falta de sensibilidad durante la fase temprana de la infección, cuando existe una baja bacteremia; o bien, cuando la bacteria se multiplica en microcolonias intracitoplasmáticas en órganos linfoides donde adquirirá mecanismos que aseguren la evasión de la respuesta inmune dentro de la célula del hospedero (Bai et al., 2017; Manasa et al. 2017; McClure et al., 2017; Tominello et al., 2019; FrancoZetina et al., 2019). Además, se han reportado falsos negativos en casos crónicos o transitorios, debido a que las mórulas suelen desaparecer cinco a ocho días después de la infección, como se ha revelado en estudios experimentales en perros y ganado ( $G a l$ et al., 2008; Stuen et al., 2013).

En México, estas enfermedades frecuentemente son subdiagnosticadas, contando con pocos estudios que determinen su prevalencia. En el 2009 en Yucatán se registró una seroprevalencia de ehrlichiosis canina del 45\% (Jiménez-Coello et al., 2009) y en otra 
investigación donde participaron 28 estados de la República Mexicana, donde se determinó la presencia de anticuerpos contra Anaplasma spp., Borrelia burgdorferi y E. canis; registrándose una alta prevalencia para E. canis (55\%) y moderada para Anaplasma spp (16,4\%), para algunos estados del noreste como Coahuila y Nuevo León (Movilla et al., 2016). Geográficamente los animales que participaron en este estudio pertenecen a la zona noreste de México; sin embargo, si comparamos la prevalencia obtenida en la zona centro de Tamaulipas para E. canis (26.8\%) con estos dos estados, sería mucho menor. No obstante, es importante mencionar que en ese estudio se

Tabla 3. Frecuencias y porcentajes de positivos y negativos a E. canis agrupados en serie roja y plaquetas

\begin{tabular}{|c|c|c|c|c|c|}
\hline \multicolumn{5}{|c|}{ Presencia de E. canis } & \multirow[t]{3}{*}{ Valor de } \\
\hline \multirow[t]{2}{*}{ Variable } & \multicolumn{2}{|c|}{ Positivo } & \multicolumn{2}{|c|}{ Negativo } & \\
\hline & Frecuencia & $\%$ & Frecuencia & $\%$ & \\
\hline Hematocrito & & & & & 0.280 \\
\hline Anemia $(<0.37 L / L)$ & 49 & 24.3 & 153 & 75.7 & \\
\hline Sin anemia $(\geq 0.37 \mathrm{~L} / \mathrm{L})$ & 54 & 29.7 & 128 & 70.3 & \\
\hline Proteínas Plasmáticas & & & & & 0.739 \\
\hline Sin hiperproteinemia $(<75 \mathrm{~g} / \mathrm{L})$ & 45 & 25.7 & 130 & 74.3 & \\
\hline Con hiperproteinemia (>75 g/L) & 58 & 27.8 & 209 & 72.2 & \\
\hline Plaquetas & & & & & 0.946 \\
\hline Trombocitopenia $\left(<180 \times 10^{9} / L\right)$ & 6 & 28.6 & 15 & 71.4 & \\
\hline Sin trombocitopenia ( $\left.\geq 180 \times 10^{9} / \mathrm{L}\right)$ & 97 & 26.7 & 266 & 73.3 & \\
\hline Leucocitos & & & & & 0.005 \\
\hline Leucopenia $\left(<6 \times 10^{9} / \mathrm{L}\right)$ & 3 & 15.8 & 16 & 84.2 & \\
\hline Normal $\left(6-17 \times 10^{9} / L\right)$ & 71 & 33.3 & 142 & 66.7 & \\
\hline Leucocitosis $\left(>17 \times 10^{9} / \mathrm{L}\right)$ & 29 & 19.1 & 123 & 80.9 & \\
\hline Monocitos & & & & & 0.060 \\
\hline Sin Monocitosis $\left(\leq 1.4 \times 10^{9} / L\right)$ & 31 & 21.1 & 116 & 78.9 & \\
\hline Monocitosis $\left(>1.4 \times 10^{9} / \mathrm{L}\right)$ & 72 & 30.4 & 165 & 69.6 & \\
\hline Linfocitos & & & & & 0.235 \\
\hline 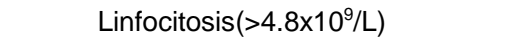 & 12 & 18.5 & 53 & 81.5 & \\
\hline Normal $\left(1.0-4.8 \times 10^{9} / \mathrm{L}\right)$ & 72 & 28.1 & 184 & 71.9 & \\
\hline Linfopenia(<1.0x109/L) & 19 & 30.2 & 44 & 69.8 & \\
\hline Neutrófilos Segmentado & & & & & 0.004 \\
\hline Neutropenia $\left(<3.0 \times 10^{9} / L\right)$ & 30 & 18.4 & 133 & 81.6 & \\
\hline Normal $\left(3.0-11.5 \times 10^{9} / \mathrm{L}\right)$ & 70 & 33.8 & 137 & 66.2 & \\
\hline 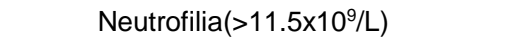 & 3 & 21.4 & 11 & 78.6 & \\
\hline Eosinófilos & & & & & 0.575 \\
\hline Sin eosinofilia $\left(<0.9 \times 10^{9} / L\right)$ & 90 & 26.2 & 253 & 73.8 & \\
\hline 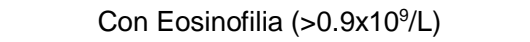 & 13 & 31.7 & 28 & 68.3 & \\
\hline
\end{tabular}


utilizaron pruebas serológicas, las cuales pueden tener el inconveniente de presentar reacción cruzada con otros microrganismos estrechamente relacionados; sobrestimando los resultados de prevalencia y planteando la necesidad de realizar estudios con técnicas moleculares que permitan evidenciar con mayor exactitud el tipo de patógeno implicado (Cetinkaya et al., 2016).

En el 2019, se realizó un estudio de detección molecular de E. canis en zonas rurales de Yucatán, encontrando un $29.26 \%$ de prevalencia (Ojeda-Chi et al., 2019), lo cual es cercano a lo reportado en este trabajo (26.8\%); pero mucho mayor comparada con la prevalencia encontrada en perros evaluados en la Comarca Lagunera (4\%) (Almazán et al., 2016).

En cuanto a las infecciones por $A$. phagocytophilum, se han diagnosticado cada vez más en animales de compañía y de granja a nivel mundial (McMahan et al., 2016). En México, A. phagocytophilum, se ha detectado en zarigüeyas y perros del estado de Campeche, con una prevalencia de 3 y $27 \%$, respectivamente (Rojero et al., 2017); sin embargo, en este trabajo ninguno de los perros evaluados resultó positivo por PCR o frotis sanguíneo. Esto no es sorprendente, ya que Ixodes spp. y Dermacentor spp., garrapatas poco frecuentes en la zona de estudio, han sido reconocidas como los vectores de mayor importancia en el ciclo de transmisión de esta bacteria, lo cual pudo contribuir a su nula presencia (Tinoco-García et al., 2009; Guzmán-Cornejo et al., 2016; Rodríguez-Vivas et al. 2019).

Los resultados observados en esta investigación muestran que $E$. canis no tiene predilección entre género, ya que dentro del grupo de los infectados los porcentajes de hembras (29.7) y machos (24.0) no fueron estadísticamente significativos ( $p>0.005)$. Esta misma variable ha sido estudiada por varios autores (Nuñez, 2003; Rodríguez-Vivas et al., 2005), encontrando resultados similares. No obstante, esto discrepa de lo registrado por otros investigadores, donde sostienen que las hembras, especialmente durante el celo preñez o parto, favorecen el riesgo de contraer infecciones por E. canis (Salazar et al., 2014; Abdelfattah et al., 2021).

En relación con los hallazgos hematológicos asociados a la presencia de ehrlichiosis y anaplasmosis canina, se ha registrado que dichas alteraciones dependerán en qué fase de la enfermedad se encuentran (Afusat et al., 2020). Durante la etapa aguda es común la presencia de anemia, la cual suele ser leve a moderada (usualmente normocítica, normocrómica, no regenerativa) (Eberts et al., 2011).

En este trabajo, la presencia de anemia no se relacionó significativamente con ninguna de las enfermedades. La trombocitopenia ha sido un hallazgo hematológico que tradicionalmente ha sido asociado a la ehrlichiosis canina (Piratae et al., 2019). Sin embargo, en este estudio la presencia de trombocitopenia $(<200,000)$ no tuvo asociación con los animales positivos a $E$. canis. Diversos estudios han reportado una asociación entre el número de plaquetas y la presencia de $E$. canis, particularmente en animales con recuento celular plaquetario inferior a 100 X10\%/L (Bulla et al., 2004; Tngsahuan et al., 
2020). Aunque en este estudio se reportaron muchos animales con presencia de anemia e hiperproteinemia, no existe asociación estadística significativa al compararlos con animales que resultaron negativos; lo anterior puede deberse a la posible presencia de otros hemoparásitos como Ehrlichia ewingii o Anaplasma platys, los cuales pueden producir grados de anemia e hiperproteinemia similares a los reportados en perros infectados con E. canis (Piratae et al., 2019).

Por otro lado, es posible que muchos de los individuos positivos a E. canis con resultados hematológicos sin alteraciones, hubieran estado en la fase subclínica de la enfermedad; esto último sería de gran importancia ya que si la enfermedad no se detecta durante esta fase podría progresar a una etapa crónica, produciendo graves daños irreversibles como trombocitopenia, leucopenia y anemia severa no regenerativa producto de una supresión de la médula ósea (Little et al., 2014).

En cuanto a la evaluación de la serie blanca, se observa que a pesar de la existencia de diferencias significativas entre los casos negativos y positivos a $E$. canis para el conteo total de leucocitos, neutrófilos y monocitos; los resultados no eran lo esperado, ya que los perros negativos resultaron con más alteraciones en estas células (ya sea que estuvieran aumentadas o disminuidas), comparados con los positivos. Estos hallazgos concuerdan con los resultados obtenidos por Asgarali y colaboradores (2012), quienes reportaron que perros con Ehrlichiosis manifestaron niveles de neutrófilos y monocitos dentro de rangos de referencia; a diferencia de los animales negativos, los cuales tuvieron un aumento significativo en dichas células. Una posible explicación de por qué muchos de los perros positivos resultaran sin alteraciones en la serie blanca, es porque tal vez estos animales pudieron encontrarse en la fase subclínica de la enfermedad, donde la mayoría son asintomáticos y no presentan alteraciones hematológicas de consideración (de Castro et al., 2004).

\section{CONCLUSIONES}

El presente estudio evidenció que las alteraciones hematológicas evaluadas en perros con signología sospechosa a Ehrlichia canis resultaron no ser específicas, ya que una gran cantidad de estos animales no se encontraban infectados. Por otro lado, muchos de los perros que sí resultaron positivos, permanecieron sin cambios aparentes en sus hemogramas, lo cual es de gran relevancia, ya que estos individuos si no se diagnostican a tiempo podrían ser reservorios para otros hospedadores incluyendo el ser humano. Además, el clínico veterinario debería considerar que estas enfermedades pueden cursar por un cuadro subclínico sin signología o con la presencia de co-infecciones que produzcan signos similares, lo cual dificultarían su diagnóstico y por ende el tratamiento adecuado. Se sugieren realizar más investigaciones que incluyan la detección de otras especies de hemoparásitos en la región, debido a su importancia como agentes potencialmente zoonóticos. 


\section{AGRADECIMIENTOS}

Al Proyecto PRODEP No. 511-6/2019.-13905 titulado "Evidencia molecular de patógenos transmitidos por garrapatas y su asociación con cambios hematológicos en caninos infectados naturalmente en Cd. Victoria, Tam., México" otorgado por el Fomento a la Generación y Aplicación Innovadora del Conocimiento (GAC) como parte del Apoyo a la reincorporación de Exbecario.

\section{LITERATURA CITADA}

ABDELFATTAH S, Alanazi AD, Alireza S, Domenico O. 2021. Seroprevalence and associated risk factors for vector-borne pathogens in dogs from Egypt. Parasit Vectors. 14:175. https://doi.org/10.1186/s13071-021-04670-0

AFUSAT JJ, Obokparo GO, Oluwafemi AA, Latifat AA. 2020. Haematology and serum chemistry in clinical canine ehrlichiosis. Vom Journal of Veterinary Science. 15(1):61-66. https://www.researchgate.net/publication/346785733

AGUIAR DM, Cavalcante GT, Pinter A, Gennari SM, Camargo LMA, Labruna MB. 2007. Prevalence of Ehrlichia canis (Rickettsiales: Anaplasmataceae) in dogs and Rhipicephalus sanguineus (Acari: Ixodidae) ticks from Brazil. Journal of Medical Entomology. 44:126-132. https://doi.org/10.1093/jmedent/41.5.126

ALLISON RW, Little SE. 2013. Diagnosis of rickettsial diseases in dogs and cats. Veterinary Clinical Pathology. 42:127-144. https://doi.org/10.1111/vcp.12040

ALMAZÁN C, Gonzalez-Alvarez VH, de Mera IGF, Cabezas-Cruz A, Rodríguez-Martínez $\mathrm{R}$, de la Fuente J. 2016. Molecular identification and characterization of Anaplasma platys and Ehrlichia canis in dogs in Mexico. Ticks and Tick-borne Diseases. 7:276-283. https://doi:10.1016/j.ttbdis.2015.11.002

ASGARALI Z, Pargass I, Adam J, Mutani A, Ezeokoli. 2012. Haematological parameters in stray dogs seropositive and seronegative to Ehrlichia canis in North Trinidad. Ticks and Tick Borne Diseases. 3(4): 207-211. https://doi.org/10.1016/j.ttbdis.2012.03.006

BAI L, Goel P, Jhambh R, Kumar P, Joshi V G. 2017. Molecular prevalence and haematobiochemical profile of canine monocytic ehrlichiosis in dogs in and around Hisar, Haryana, India. J Parasit Dis. 41(3): 647-54. https://doi.org/10.1007/s12639- 016-0860-8

BATTILANI M, De Arcangeli S, Balboni A, Dondi F. 2017. Genetic diversity and molecular epidemiology of Anaplasma. Infection Genetics and Evolution. 49:195-211. https://doi.org/10.1016/j.meegid.2017

BEUGNET F, Chalvet-Monfray K. 2013. Impact of climate change in the epidemiology of vector-borne diseases in domestic carnivores. Comp Immunol Microbiol Infect Dis. 36:559-566. https://doi.org/10.1016/j.cimid.2013.07.003 
BHADESIYA CM, Modi DV. 2015. Correlation of epidemiology of Rhipicephalous sanguineus and canine ehrlichiosis in nine different localities of middle Gujarat. International Agricultural Science and Veterinary Medicine. 3(1): 2320-3730. http://citeseerx.ist.psu.edu/viewdoc/download?doi=10.1.1.740.420\&rep=rep1\&type=pdf

BULLA C, Kiomi R, Pessoa J, Trinca LA, Souza R, Wiedmeyer CE. 2004. The relationship between the degree of thrombocytopenia and infection with Ehrlichia canis in an endemic area. Veterinary Research. 35:141-146. https://doi.org/10.1051/VETRES:2003038

CABEZAS-CRUZ A, Allain E, Ahmad AS, Saeed MA, Rashid I, Ashraf K, Estrada-Peña A. 2019. Low genetic diversity of Ehrlichia canis associated with high co-infection rates in $\begin{array}{llll}\text { Rhipicephalus sanguineus (sl). Parasites \& Vectors. 12:12. } & \text { \& }\end{array}$ https://doi.org/10.1186/s13071-018-3194-9

CETINKAYA H, Matur E, Akyazi I, Ekiz EE, Aydin L, Toparlak M. 2016. Serological and molecular investigation of Ehrlichia spp. and Anaplasma spp. in ticks and blood of dogs, in the Thrace Region of Turkey. Ticks and Tickborne Diseases. 7:706-714. https://doi.org/10.1016/j.ttbdis.2016.02.021

DE CASTRO MB, Machado RZ, de Aquino LP, Alessi AC, Costa MT. 2004. Experimental acute canine monocytic ehrlichiosis: clinicopathological and immunopathological findings. Vet Parasitol. 119:73-86. https://doi.org/10.1016/J.VETPAR.2003.10.012

DE LA FUENTE J, Antunes S, Bonnet S, Cabezas-Cruz A, Domingos AG, Estrada-Peña $A$, et al. 2017. Tick-pathogen interactions and vector competence: identification of molecular drivers for tick-borne diseases. Frontiers in cellular and infection microbiology. 7:114. https://doi.org/10.3389/fcimb.2017.00114

DUMLER JS, Barbet AF, Bekker CP, Dasch GA, Palmer GH, Ray SC, Rurangirwa FR. 2001. Reorganization of genera in the families Rickettsiaceae and Anaplasmataceae in the order Rickettsiales: unification of some species of Ehrlichia with Anaplasma, Cowdria with Ehrlichia and Ehrlichia with Neorickettsia, descriptions of six new species combinations and designation of Ehrlichia equi and HGE agent as subjective synonyms of Ehrlichia phagocytophila. International Journal of Systematic and Evolutionary Microbiology. 51(6):2145-2165. https://doi.org/10.1099/00207713-51-6-2145

EBERTS MD, Vissotto de Paiva Diniz PP, Beall MJ, Stillman BA, Chandrashekar R, Breitschwerdt EB. 2011. Typical and atypical manifestations of Anaplasma phagocytophilum infection in dogs. Journal of the American Animal Hospital Association. 47:86-94. https://doi.org/10.5326/JAAHA-MS-5578

FARHAN AA. 2015. Anaplasma marginale and Anaplasma phagocytophilum: Rickettsiales pathogens of veterinary and public health significance. Parasitol Res. 114(11):3941-57. https://doi.org/10.1007/s00436-015-4698-2 
FRANCO-ZETINA M, Adame-Gallegos J, Dzul-Rosado K. 2019. Effectivity of diagnostic methods for the detection of human and canine monocytic ehrlichiosis. Rev. Chilena Infectol. 36(5):650-655. https://doi.org/10.4067/S0716-10182019000500650

GAL A, Loeb E, Yisaschar-Mekuzas Y, Baneth G. 2008. Detection of Ehrlichia canis by PCR in different tissues obtained during necropsy from dogs surveyed for naturally occurring canine monocytic ehrlichiosis. Veterinary Journal. 175:212-217. https://doi.org/10.1016/J.TVJL.2007.01.013

GUZMÁN-CORNEJO C, Robbins RG, Guglielmone AA, Montiel-Parra G, Rivas G, Pérez TM. 2016. The Dermacentor (Acari, Ixodida, Ixodidae) of Mexico: hosts, geographical distribution and new records. ZooKeys. 569:1-22. https://doi.org/10.3897/zookeys.569.7221

HAPPI AN, Toepp AJ, Ugwu CA, Petersen CA, Sykes JE. 2018. Detection and identification of blood-borne infections in dogs in Nigeria using light microscopy and the polymerase chain reaction. Vet Parasitol Reg Stud Reports. 11: 5560. https://doi.org/10.1016/j.vprsr.2017.12.002

HARRUS S, Waner T. 2011. Diagnosis of canine monocytotropic ehrlichiosis (Ehrlichia canis): an overview. The Veterinary Journal. 187:292-296. https://doi.org/10.1016/j.tvjl.2010.02.001

IRWIN PJ. 2014. It shouldn't happen to a dog ... or a veterinarian: clinical paradigms for canine vector-borne diseases. Trends Parasitology 30:104-12. https://doi.org/10.1016/j.pt.2013.12.001

ISMAIL N, McBride JW. 2017. Tick-borne emerging infections: ehrlichiosis and anaplasmosis. Clinics in Laboratory Medicine. 37:317-340. https://doi.org/10.1016/j.cll.2017.01.006

JIMÉNEZ-COELLO M, Pérez-Osorio C, Vado-Solís I, Rodríguez-Buenfil JC, OrtegaPacheco A. 2009. Serological survey of Ehrlichia canis in stray dogs from Yucatan, Mexico, using two different diagnostic tests. Vector Borne Zoonotic Diseases. 9:209-212. https://doi.org/10.1089/vbz.2008.0039

LITTLE E, Beall MJ, Bowman DD, Chandrashekar R, Stamaris J. 2014. Canine infection with Dirofilaria immitis, Borrelia burgdorferi, Anaplasma spp., and Ehrlichia spp. in the United States, 2010-2012. Parasites \& Vectors. 7:1-9. https://parasitesandvectors.biomedcentral.com/articles/10.1186/1756-3305-7-257

MAGGI RG, Krämer F. 2019. A review on the occurrence of companion vector-borne diseases in pet animals in Latin America. Parasitic Vectors. 12:145. doi: 10.1186/s13071019-3407-x 
MANASA RK, Pritpal SD, Lachhman DS, Baljinder KB, and Sanjeev KU. 2017. Clinical and hematobiochemical response in canine monocytic ehrlichiosis seropositive dogs of Punjab. Veterinary World. 10(2): 255-261. https://doi.org/10.14202/vetworld.2017.255261

MCCLURE EE, Chávez ASO, Shaw DK, Carlyon JA, Ganta RR, Noh SM, Wood DO, Ba voil PMA, Brayton KA, Martinez JJ, McBride JW, Valdivia RH, Munderloh UG, Pedra JHF. 2017. Engineering of obligate intracellular bacteria: progress, challenges and paradigms. Nat Rev Microbiol. 15:544-558. https://doi.org/10.1038/nrmicro.2017.59

MCMAHAN CS, Wang D, Beall MJ, Bowman DD, Little SE, Pithua PO, Julia L, Sharp JL, Stich RW, Yabsley MJ, Lund RB. 2016. Factors associated with Anaplasma spp. seroprevalence among dogs in the United States. Parasites \& Vectors. 9:169. https://doi.org/10.1186/s13071-016-1431-7

MOVILLA R, García C, Siebert S, Roura X. 2016. Countrywide serological evaluation of canine prevalence for Anaplasma spp., Borrelia burgdorferi (sensu lato), Dirofilaria immitis and Ehrlichia canis in Mexico. Parasites \& Vectors. 9:421. https://doi.org/10.1186/s13071-016-1686-z

NÚÑEZ OL. 2003. Estudio de la seroprevalencia de Ehrlichia canis en México. Asociación Méxicana de Médicos Veterinarios Especialistas en Pequeñas Especies. 14:83-85. https://www.researchgate.net/publication/291003826

OJEDA-CHI MM, Rodriguez-Vivas RI, Esteve-Gasent MD, Pérez de León A, Modarelli JJ, Villegas-Perez SL. 2019. Ehrlichia canis in dogs of Mexico: Prevalence, incidence, coinfection and factors associated. Comparative Immunology, Microbiology and Infectious Diseases. 67:101351. https://doi.org/10.1016/j.cimid.2019.101351

PAROLA P, Paddock CD, Socolovschi C, Labruna MB, Mediannikov O, Kernif T, et al. 2013. Update on tick-borne rickettsioses around the world: a geographic approach. Clin Microbiol Rev. 26:657-702. https://doi.org/10.1128/CMR.00032-13

PIRATAE S, Senawong P, Chalermchat P, Harnarsa W, Sae-chue B. 2019. Molecular evidence of Ehrlichia canis and Anaplasma platys and the association of infections with hematological responses in naturally infected dogs in Kalasin. Thailand. Veterinary World. 12:131. https://doi.org/10.14202/vetworld.2019.131-135

RODRÍGUEZ-VIVAS RI, Albornoz REF, Bolio GME. 2005. Ehrlichia canis in dogs in Yucatan, Mexico: seroprevalence, prevalence of infection and associated factors. Veterinary Parasitology. 127:75-79. https://doi.org/10.1016/J.VETPAR.2004.08.022

RODRÍGUEZ-VIVAS RI, Ojeda-Chi MM, Bolio-González ME, Rosado-Aguilar JA. 2019. Las garrapatas como vectores de enfermedades zoonóticas en México. Bioagrociencias. 12(1):19-26. https://www.revista.ccba.uady.mx/ojs/index.php/BAC/article/view/2993 
ROJERO-VÁZQUEZ R, Gordillo-Pérez G, Weber M. 2017. Infection of Anaplasma phagocytophilum and Ehrlichia spp. in opossums and dogs in Campeche, Mexico: the role of tick infestation. Frontiers in Ecology Evolution. 5:161. https://doi.org/10.3389/fevo.2017.00161

SALAZAR H, Edwin F, Buriticá EF, Echeverry DF, Barbosa I. 2014. Seroprevalencia de Ehrlichia canis y su relación con algunos parámetros clínicos y hematológicos en caninos admitidos en clínicas veterinarias de la ciudad de Ibagué (Colombia). Revista Colombiana de Ciencia Animal. 7 (1):56-63.

http://revistas.ut.edu.co/index.php/ciencianimal/article/view/542

STICH RW, Rikihisa Y, Ewing SA, Needham GR, Grover DL, Jittapalapong S. 2002. Detection of Ehrlichia canis in canine carrier blood and in individual experimentally infected ticks with a p30-based PCR assay. Journal of Clinical Microbiology. 40:540-546. https://doi.org/10.1128/JCM.40.2.540-546.2002

STUEN S, Granquist EG. Silaghi C. 2013. Anaplasma phagocytophilum a widespread multi-host pathogen with highly adaptive strategies. Frontiers in Cellular and Infection Microbiology. 3:31. https://doi.org/10.3389/fcimb.2013.00031

TINOCO-GRACIA L, Quiroz-Romero H, Quintero-Martinez MT, Renteria-Evangelista TB, Gonzalez-Medina Y, Barreras-Serrano A, et al. 2009. Prevalence of Rhipicephalus sanguineus ticks on dogs in a region on the Mexico-USA border. Veterinary Record. 164:59-61. https://doi.org/10.1136/vr.164.2.59

TOMINELLO TR, Oliveira ERA, Hussain SS, Wells AEJ, Golden B, Ismail N. 2019. Emerging roles of autophagy and inflammasome in ehrlichiosis. Front Immunol. 10:1011. https://doi.org/10.3389/fimmu.2019.01011

TNGSAHUAN S, Chethanond U, Wasiksiri S, Saechan V, Thongtako W, Musikacharoen T. 2020. Hematological profile of blood parasitic infected dogs in Southern Thailand. Vet World. 13(11): 2388-2394. https://doi.org/10.14202/vetworld.2020.2388-2394

VIEIRA RFDC, Vieira TSWJ, Nascimento, DDAG, Martins TF, Krawczak FS, Labruna MB, Vidotto O. 2013. Serological survey of Ehrlichia species in dogs, horses, and humans: zoonotic scenery in a rural settlement from southern Brazil. Revista do Instituto de Medicina Tropical de São Paulo. 55:335-340. https://doi.org/10.1590/S003646652013000500007

WAYNE WD, Chad LC. 2013. Biostatistics: A Foundation for Analysis in the Health Sciences. 10th Edition Wiley. Pp. 191. ISBN 978-1-118-30279-8.

YOUSEFI A, Chaechi Nosrati MR, Golmohammadi A, Azami S. 2019. Molecular Detection of Anaplasma Phagocytophilum as a Zoonotic Agent in Owned and Stray Dogs in Tehran, Iran. Archives of Razi Institute. 74:33-38. https://doi.org/10.22092/ARI.2018.114893.1142 